\title{
Volume-preserving diffeomorphism as nonabelian higher-rank gauge symmetry
}

\author{
Yi-Hsien Du, ${ }^{1}$ Umang Mehta, ${ }^{1}$ Dung Xuan Nguyen, ${ }^{2}$ and Dam Thanh Son ${ }^{1}$ \\ ${ }^{1}$ Kadanoff Center for Theoretical Physics, \\ University of Chicago, Illinois 60637, USA \\ ${ }^{2}$ Brown Theoretical Physics Center, Brown University, Providence, RI 02912, USA
}

We propose nonabelian higher-rank gauge theories in $2+1 \mathrm{D}$ and $3+1 \mathrm{D}$. The gauge group is constructed from the volume-preserving diffeomorphisms of space. We show that the intriguing physics of the lowest Landau level (LLL) limit can be interpreted as the consequences of the symmetry. We derive the renowned Girvin-MacDonaldPlatzman (GMP) algebra as well as the topological Wen-Zee term within our formalism. Using the gauge symmetry in $2+1 \mathrm{D}$, we derive the LLL effective action of vortex crystal in rotating Bose gas as well as Wigner crystal of electron in applied magnetic field. We show that the non-linear sigma models of ferromagnet in $2+1 \mathrm{D}$ and $3+1 \mathrm{D}$ exhibit the higher-rank gauge symmetries that we introduce in this paper. We interpret the fractonic behavior of the excitations on the lowest Landau level and of skyrmions in ferromagnets as the consequence of the higher-rank gauge symmetry.

\section{INTRODUCTION}

Recently, considerable interest has been drawn to "higher-rank gauge theories," i.e., theories where the gauge potential is not a one-form, but a tensor of higher rank [1-14]. The physical motivation of the higher-rank gauge theories is the discovery of a new class of topological matter known as fractons [15-19], one feature of which is the existence of restricted mobile excitations. The excitations either cannot move at all or can only move in lowerdimensional sub-spaces, while composites of elementary excitations can move freely. The restricted mobility of the fractonic excitations can be interpreted as the consequence of the higher rank theories' conservation laws. In particular, the tensor Gauss's law leads to the conservation of not only the electric charge, but also electric dipole moment and, in some cases, higher moments of the charge distribution [1, 2, 19]. This has the physical effect of rendering the electric charge immobile but leaving the dipoles mobile or partially mobile. One representative example is the so-called "traceless scalar charge theory," in which the electric charges are immobile and the electric dipole can move only in the direction perpendicular to the dipole moment [2]. The higher-rank gauge theories have also been applied to describe defects in solids [20-24], supersolids [25], superfluid vortices [26, 27], smectics [5, 27, 28], and other systems.

All the higher-rank gauge symmetries that were mentioned in above physical models are abelian. There were attempts to generalize these symmetries to nonabelian symmetries 
[6, 29-31], however a physical system that realizes those symmetries has not been explicily proposed. In this paper we propose the non-abelian higher-rank symmetry theories for physical condensed matter systems. We reformulate the tensor gauge transformation in the traceless scalar charge theory in $2+1 \mathrm{D}[1,2]$. We show that the gauge transformation is nothing but the volume-preserving diffeomorphism (VPD) in the linearized form. We then construct the nonlinear higher-rank gauge theory with VPDs as the symmetry group. The nonabelian nature of the gauge symmetry has a nontrivial consequence - the charge density operators at different positions do not commute. Instead, they form the long-wavelength limit of the Girvin-MacDonald-Platzman (GMP) algebra [32-34], which reveals a connection to the lowest Landau level (LLL).

In fact, it is easy to notice noticed that the many feature of the physics on the LLL, bears close resemblance to that of the field-theory models with higher rank symmetries [35]: for example, electric charges are pinned to one place by the large magnetic field, and neutral excitations (e.g., the composite fermion in the half-filled Landau level [36]) carrying AN electric dipole moment and can move in the direction perpendicular to the direction of motion. In this paper we show that this resemblance is not accidental; in fact, the nonlinear higher-rank symmetry is realized as a symmetry of the problem of charged particles on the LLL. The relation between the dipole moment and momentum of an excitation in models with higher-rank symmetry was noticed in Refs. [13, 26].

We also will present several physical systems that enjoy the non-abelian higher-rank symmetry. In 2+1D systems, the symmetry originates from the lowest Landau level limit, where the volume-preserving is the restriction for transformations that preserve the background magnetic field. In addition to the derivation of the GMP algebra, we show draw a connection between the topological Wen-Zee term [37] with the Chern-Simons term in a higher-rank gauge theory. Furthermore, we will use the gauge symmetry formalism to derive the effective theories of the Wigner crystal of electrons in strong a magnetic field, of the vortex crystal in a rotating Bose gas.

Finally, we find that the nonlinear sigma model describing ferromagnetism in $2+1 \mathrm{D}$ and $3+1 \mathrm{D}$ also exhibits the global higher-rank symmetry. The higher rank gauge symmetry provides a new interpretation of the conservation of multipole moments in ferromagnets [38], and of the close resemblence between the behaviors of skyrmions in ferromagnets and of charged particles in a magnetic field [39].

\section{REVIEW OF THE TRACELESS SCALAR CHARGE THEORY}

For self-consistency, in this Section, we will review the symmetric tensor gauge theory proposed by Pretko [1, 2]. We consider a higher-rank gauge theory called "traceless scalar charge theory", where gauge potential is a symmetric rank-2 tensor $A_{i j}: A_{i j}=A_{j i}$. Its conjugate momentum is the electric field $E_{i j}$. They satisfy the canonical commutation 
relation

$$
\left[E_{i j}(\mathbf{x}), A_{k l}(\mathbf{y})\right]=i\left(\delta_{i k} \delta_{j l}+\delta_{i l} \delta_{j k}\right) \delta(\mathbf{x}-\mathbf{y}) .
$$

One imposes the Gauss law and the traceless constraint:

$$
\begin{gathered}
\partial_{i} \partial_{j} E_{i j}=\rho, \\
E \equiv E_{i i}=0,
\end{gathered}
$$

which lead to the conservation of the following charges

$$
\int d \mathbf{x} \rho(\mathbf{x}), \quad \int d \mathbf{x} \mathbf{x} \rho(\mathbf{x}), \quad \int d \mathbf{x} x^{2} \rho(\mathbf{x}) .
$$

The conservations in Eq. (4) imply that a charge cannot move, and a dipole can move only along the direction perpendicular to the dipole moment [1].

The constraints (2) and (3) generate the gauge tranformations on the gauge potential

$$
\begin{aligned}
& A_{i j} \rightarrow A_{i j}+\partial_{i} \partial_{j} \lambda, \\
& A_{i j} \rightarrow A_{i j}+\delta_{i j} \mu .
\end{aligned}
$$

For our purpose, it is convenient to use the second gauge invariance (6) to explicitly fix the gauge $A \equiv A_{i i}=0$ and eliminate the trace of $A_{i j}$ from the set of dynamical degrees of freedom. Since the two constraints $E=0$ and $A=0$ do not commute according to the commutation relation (1), following Dirac one should modify the commutators, replacing them by the Dirac brackets [40], which in our case is

$$
\left[O_{1}, O_{2}\right] \rightarrow\left[O_{1}, O_{2}\right]_{\mathrm{D}}=\left[O_{1}, O_{2}\right]+\left[O_{1}, E\right][E, A]^{-1}\left[A, O_{2}\right]-\left[O_{1}, A\right][E, A]^{-1}\left[E, O_{2}\right]
$$

The new commutator is then

$$
\left[E_{i j}(\mathbf{x}), A_{k l}(\mathbf{y})\right]=i\left(\delta_{i k} \delta_{j l}+\delta_{i l} \delta_{j k}-\frac{2}{d} \delta_{i j} \delta_{k l}\right) \delta(\mathbf{x}-\mathbf{y}) .
$$

The Gauss constraint $\partial_{i} \partial_{j} E_{i j}=\rho$ generates now the gauge transformation

$$
A_{i j} \rightarrow A_{i j}+\partial_{i} \partial_{j} \lambda-\frac{1}{d} \delta_{i j} \partial^{2} \lambda, \quad \partial^{2} \equiv \partial^{k} \partial_{k} .
$$

To construct a gauge-invariant Lagrangian, we introduce the field strengths. The electric field

$$
E_{i j}=\partial_{i} \partial_{j} A_{0}-\frac{1}{d} \delta_{i j} \partial^{2} A_{0}-\partial_{t} A_{i j}
$$

is obviously gauge invariant. One notices that

$$
\omega_{i}=-\partial_{j} A_{i j}
$$

transforms as a $U(1)$ vector potential,

$$
\omega_{i} \rightarrow \omega_{i}-\partial_{i} \tilde{\lambda}, \quad \tilde{\lambda}=\left(1-\frac{1}{d}\right) \partial^{2} \lambda,
$$


using which one can define the magnetic field,

$$
H_{i j}=\partial_{i} \omega_{j}-\partial_{j} \omega_{i}=-\partial_{i} \partial_{k} A_{j k}+\partial_{j} \partial_{k} A_{i k}
$$

that manifests the gauge invariant. The simplest Lagrangian for the gauge field is then the one of the "Maxwell theory,"

$$
L=c_{1} E_{i j}^{2}-c_{2} H_{i j} H_{i j}
$$

However, as noticed in Ref. [41], in $(2+1) \mathrm{D}$, another possible term in the Lagrangian is the Chern-Simons term which, up to an overall coefficient, reads

$$
L_{\mathrm{CS}}=\varepsilon^{i j}\left(A_{0} H_{i j}-A_{i k} \dot{A}_{j k}\right)
$$

As for a Chern-Simons term, the Lagrangian density is gauge-invariant only up to a total derivative. This term is more relevant than the Maxwell term. The higher-rank ChernSimons theory in 3 spatial dimensions was also discussed previously in Ref. [42].

Note that one does not have to require the theory to contain dynamical gauge fields in order to have the conserve quantities (4). A theory coupled to a background gauge fields $\left(A_{0}, A_{i j}\right)$ and is invariant under the gauge symmetry under which the gauge fields transform as

$$
A_{0} \rightarrow A_{0}+\dot{\lambda}, \quad A_{i j}+\partial_{i} \partial_{j} \lambda-\frac{1}{d} \delta_{i j} \partial^{2} \lambda
$$

will have the conservation law

$$
\partial_{t} \rho-\partial_{i} \partial_{j} J_{i j}=0
$$

where $\rho$ and $J_{i j}$ are the operators that couple to $A_{0}$ and $A_{i j}$, respectively, and $J_{i i}=0$. This is sufficient to derive the conservation of the quantities (4). In fact, in most of this paper we will consider theories coupled to background nondynamical gauge fields.

\section{NONLINEAR HIGHER-RANKED SYMMETRY}

\section{A. Traceless scalar charge theory in $(2+1) D$ as a theory of linearized gravity}

We now show that the theory presented in the previous Section can be interpreted as a theory of linearized gravity, and the higher rank symmetry as the linearized version of VPD. Instead of $A_{i j}$ we introduce an equivalent field $h_{i j}$ defined as

$$
h_{i j}=-\ell^{2}\left(\varepsilon_{i k} A_{j k}+\varepsilon_{j k} A_{i k}\right),
$$

where $\ell$ is some constant of the dimension of length ${ }^{1}$. Note that $h_{i j}$ is also symmetric and traceless. The gauge transformation for $h_{i j}$ is inherited from (16)

$$
h_{i j} \rightarrow h_{i j}-\ell^{2}\left(\varepsilon_{i k} \partial_{j} \partial_{k}+\varepsilon_{j k} \partial_{i} \partial_{k}\right) \lambda
$$

\footnotetext{
1 We assume $A_{0}$ has dimension 1 and $A_{i j}$ is of dimension 2 , so $h_{i j}$ is dimensionless.
} 
If we define

$$
\xi^{i}=\ell^{2} \varepsilon^{i k} \partial_{k} \lambda,
$$

then the transformation law of $h_{i j}$ can be reformulated as

$$
h_{i j} \rightarrow h_{i j}-\partial_{i} \xi_{j}-\partial_{j} \xi_{i} .
$$

The transformation (21) is nothing but the transformation of the metric under volumepreserving (or in 2D, area-preserving) diffeomorphism $x^{i} \rightarrow x^{i}+\xi^{i}$, since $\partial_{i} \xi^{i}=0$ due to the definition (20). The connection between a higher-rank gauge theory and a linearized gravity theory was proposed previously in [43].

\section{B. Nonlinear higher-rank symmetry}

The fact that the gauge symmetry resembles the transformation law of a metric under VPDs allows one to devise a nonlinear version of the gauge symmetry. Namely, in our nonlinear theory, instead of a gauge field $h_{i j}$ (or $A_{i j}$ for that matter), the degree of freedom is the metric $g_{i j}$. The tracelessness of $h_{i j}$ translates into the statement that the metric is unimodular: $\operatorname{det} g=1$. The linear theory is restored when one expands the metric around the flat metric: $g_{i j}=\delta_{i j}+h_{i j}+O\left(h^{2}\right)$.

Under an infinitesimal VPD $x^{i} \rightarrow x^{i}+\xi^{i}=x^{i}+\ell^{2} \varepsilon^{i j} \partial_{j} \lambda$, the metric transforms as

$$
\delta_{\lambda} g_{i j}=-\xi^{k} \partial_{k} g_{i j}-g_{k j} \partial_{i} \xi^{k}-g_{i k} \partial_{j} \xi^{k}=-\ell^{2} \varepsilon^{k l}\left(\partial_{k} g_{i j}+g_{k j} \partial_{i}+g_{i k} \partial_{j}\right) \partial_{l} \lambda .
$$

Now we need to write down the nonlinear version of the transformation laws for $A_{0}$. One notices that the VPDs do not commute: from Eq. (22) one reads

$$
\left[\delta_{\alpha}, \delta_{\beta}\right]=\delta_{[\alpha, \beta]},
$$

with $[\alpha, \beta]=\ell^{2} \varepsilon^{i j} \partial_{i} \alpha \partial_{j} \beta$. This means that our gauge symmetry is nonabelian; in this paper we will use "nonlinear" and "nonabelian" interchangeably. The transformation of $A_{0}$ must satisfy the commutation relation (23). One can check that this can be accomplished by the following simple modification

$$
\delta_{\lambda} A_{0}=\partial_{t} \lambda-\xi^{k} \partial_{k} A_{0}=\partial_{t} \lambda-\ell^{2} \varepsilon^{k l} \partial_{k} A_{0} \partial_{l} \lambda .
$$

Equations (24) and (22) give the transformation laws of a nonlinear higer-rank symmetry, which we collect here for convenience:

$$
\begin{aligned}
& \delta_{\lambda} A_{0}=\partial_{t} \lambda-\ell^{2} \varepsilon^{k l} \partial_{k} A_{0} \partial_{l} \lambda, \\
& \delta_{\lambda} g_{i j}=-\ell^{2} \varepsilon^{k l}\left(\partial_{k} g_{i j}+g_{k j} \partial_{i}+g_{i k} \partial_{j}\right) \partial_{l} \lambda .
\end{aligned}
$$

A nonlinear transformation similar to Eq. (26) was considered in Ref. [14] within a dynamical gauge model of the traceless scalar charge theory. One can derive the Ward identity from 
Eqs. (25), (26). Let us define the charge density $\rho$ and the stress tensor $T^{i j}$ by varying of the logarithm of the partition function

$$
\delta \ln Z=\int d^{3} x\left(\rho \delta A_{0}+\frac{1}{2} T^{i j} \delta h_{i j}\right)
$$

The Ward identity is then

$$
\dot{\rho}-\ell^{2} \varepsilon^{k l} \partial_{l}\left[\rho \partial_{k} A_{0}+\frac{1}{2} T^{i j} \partial_{k} g_{i j}+\partial_{i}\left(T^{i j} g_{j k}\right)\right]=0
$$

In the presence of the background field only the total charge is conserved, but not the higher multipoles in (4).

Since the total charge is conserved, it is possible to introduce a vector potential $A_{i}$ so that the theory is invariant under the usual $U(1)$ gauge symmetry $A_{\mu} \rightarrow A_{\mu}+\partial_{\mu} \alpha$. In this case $A_{0}$ plays a double role: it is the temporal component of a $\mathrm{U}(1)$ gauge field $\left(A_{0}, A_{i}\right)$, and also as the scalar component of the gauge potential of a higher-spin symmetry, $\left(A_{0}, g_{i j}\right)$. The two sets of gauge potentials share one scalar component, see Fig. 1. We will see an example when we consider ferromagnets (Sec. VID).

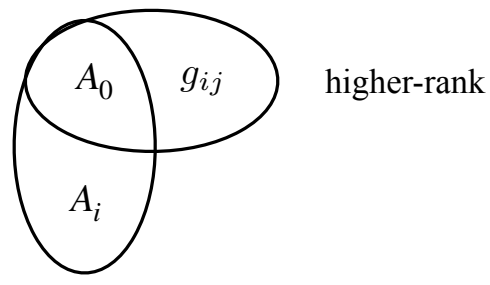

$U(1)$

FIG. 1. $A_{0}$ is shared by two sets of gauge potentials.

The nonabelian nature of the gauge symmetry, in some cases, allows us to derive the algebra satisfied by the charge density of the matter coupled to the gauge field. Imagine that the action $S_{\mathrm{m}}\left(\psi, A_{0}, g_{i j}\right)$ describing the coupling of the matter fields $\psi$ with the gauge fields $\left(A_{0}, g_{i j}\right)$ does not contain the time derivatives of any fields, $A_{0}, g_{i j}$, or $\psi$. In this case, if one promotes the gauge fields to dynamical fields by adding to the action a pure gauge action $S_{\mathrm{g}}$,

$$
S=S_{\mathrm{g}}\left[A_{0}, g_{i j}\right]+S_{\mathrm{m}}\left[\psi, A_{0}, g_{i j}\right]
$$

then upon quantization the canonical commutation relations in the gauge sector are set by $S_{\mathrm{g}}$ and in the matter sector by $S_{\mathrm{m}}$. The left-hand side of the Gauss constraint,

$$
\frac{\delta S_{\mathrm{g}}}{\delta A_{0}}+\frac{\delta S_{\mathrm{m}}}{\delta A_{0}}=0
$$

is the generator that generates gauge transformations. In particular, in the matter sector, the commutator of the charge density $\rho(\mathbf{x})$ with any matter field $O(\mathbf{x})$ will give the change 
of $O$ under infinitesimal gauge tranformation:

$$
\left[\int d \mathbf{y} \lambda(\mathbf{y}) \rho(\mathbf{y}), O(\mathbf{x})\right]=i \delta_{\lambda} O(\mathbf{x}) .
$$

But diffeomorphisms do not commute, so we conclude that in our theory the charge density at different points do not commute. We find

$$
[\rho(\mathbf{x}), \rho(\mathbf{y})]=i \ell^{2} \varepsilon^{i j} \partial_{i} \rho(\mathbf{x}) \partial_{j} \delta(\mathbf{x}-\mathbf{y}) .
$$

Here we recover the long-wavelength version of the Girvin-MacDonald-Platzman (GMP) algebra [32] (or the $w_{\infty}$ algebra), which suggests that the symmetry described here is related to the physics of the LLL.

\section{CONNECTION TO QUANTUM HALL EFFECT}

To establish the connection with the physics of the LLL, we recall the symmetry of the problem. A system of particles interacting with an electromagnetic field can also be put in curved space:

$$
S=\int d t d \mathbf{x} \sqrt{g}\left(\frac{i}{2} \psi^{\dagger} \stackrel{\leftrightarrow}{\partial}_{t} \psi+A_{0} \psi^{\dagger} \psi-\frac{g_{i j}}{2 m} D_{i} \psi^{\dagger} D_{j} \psi+\cdots\right),
$$

where $\cdots$ include interaction terms. (Strictly speaking, the discussion here corresponds to the $g=2, s=1$ version of the LLL symmetry [44].) One can check that the classical action is invariant with respect to time-dependent spatial diffeomorphisms (i.e., all diffeomorphism transformations which preserve the time slices) [44, 45]:

$$
\begin{aligned}
\delta A_{0} & =-\xi^{k} \partial_{k} A_{0}-A_{k} \dot{\xi}^{k} \\
\delta A_{i} & =-\xi^{k} \partial_{k} A_{i}-A_{k} \partial_{i} \xi^{k}-m g_{i k} \dot{\xi}^{k} \\
\delta g_{i j} & =-\xi^{k} \partial_{k} g_{i j}-g_{i k} \partial_{j} \xi^{k}-g_{i k} \partial_{j} \xi^{k} .
\end{aligned}
$$

The LLL limit corresponds to taking $m \rightarrow 0$. The term proportional to $m$ disappears from the transformation law for $A_{i}$; now $A_{\mu}$ simply transforms like a one-form under spatial diffs:

$$
\delta A_{\mu}=-\xi^{k} \partial_{k} A_{\mu}-A_{\lambda} \partial_{\mu} \xi^{\lambda}, \quad \xi^{\lambda}=\left(0, \xi^{i}\right) .
$$

The metric $g_{i j}$ also transforms like a covariant tensor. The nontrivial feature of the states on the LLL that sets it apart from other states in a magnetic field is that, although a time-varying diffeomorphism generates the $g_{0 i}$ components of the metric tensor from $g_{i j}$ : $\delta g_{0 i}=\cdots+g_{i j} \dot{\xi}^{j}$, the partition function of the theory does not depend at all on $g_{0 i}$.

The fractional quantum Hall effect exists in a finite magnetic field $B=\partial_{1} A_{2}-\partial_{2} A_{1}$. If one is not interested in computing the electric current by varying the partition funciton with respect to $A_{i}$, then one can assume that $A_{i}$ has some fixed value, for example, $A_{i}=-\frac{1}{2} B \varepsilon_{i j} x^{j}$, 
and only consider $A_{0}$ and $g_{i j}$ as external backgrounds. Then it is natural to ask if the partition function of the theory is symmetric under any gauge transformation that touches only $A_{0}$ and $g_{i j}$, and explore the Ward-Takahashi identities that follow. To keep $B$ unchanged we needs to restrict ourselves to VPDs. These correspond to $\xi^{k}=\ell^{2} \varepsilon^{k l} \partial_{l} \lambda$, where we chose $\ell$ to be the magnetic length $\ell=1 / \sqrt{B}$. For VPDs, the change of the spatial components of gauge potential $A_{i}$,

$$
\begin{array}{r}
\delta A_{i}=-\ell^{2} \varepsilon^{k l} \partial_{l} \lambda \partial_{k} A_{i}-\ell^{2} A_{k} \varepsilon^{k l} \partial_{i} \partial_{l} \lambda=-\ell^{2} \varepsilon^{k l} \partial_{l} \lambda\left(\partial_{k} A_{i}-\partial_{i} A_{k}\right)-\ell^{2} \partial_{i}\left(\varepsilon^{k l} A_{k} \partial_{l} \lambda\right) \\
=-\partial_{i}\left(\lambda+\ell^{2} \varepsilon^{k l} A_{k} \partial_{l} \lambda\right)
\end{array}
$$

can be compensated by a gauge transformation $A_{\mu} \rightarrow A_{\mu}+\partial_{\mu} \alpha$ with $\alpha=\lambda+\ell^{2} \varepsilon^{k l} A_{k} \partial_{l} \lambda$. Under this combination of coordinates and gauge transformations, $A_{0}$ transforms as

$$
\delta A_{0}=-\ell^{2} \varepsilon^{k l} \partial_{l} \lambda \partial_{k} A_{0}-\ell^{2} A_{k} \partial_{t}\left(\varepsilon^{k l} \partial_{l} \lambda\right)+\dot{\lambda}+\ell^{2} \partial_{t}\left(\varepsilon^{k l} A_{k} \partial_{l} \lambda\right)=\dot{\lambda}-\ell^{2} \varepsilon^{k l} \partial_{k} A_{0} \partial_{l} \lambda .
$$

We see that the transformation law for $A_{0}$ has exactly the form that we have postulated in Eq. (24). The metric, of course, transforms as in Eq. (22).

\section{A. Higher-rank symmetry in the lowest Landau level limit}

Within the context of the LLL, it is possible to give an intuitive interpretation of the higher-rank conservation law. We write down the current conservation

$$
\frac{\partial \rho}{\partial t}+\nabla \cdot \mathbf{j}=0
$$

and the law of conservation of momentum,

$$
\frac{\partial \pi_{i}}{\partial t}+\partial_{j} T_{i j}=E_{i} \rho+\varepsilon_{i k} j_{k} B
$$

where $\pi_{i}$ is the momentum density. In a Galilean-invariant theory with particles of mass $m$, the momentum density is proportional to the particle number flux $\pi_{i}=m j_{i}$, and vanishes in the LLL limit $m \rightarrow 0$. Now the conserivation of momentum becomes simply the equation of balance of force, which in the absence of the electric field simply reads

$$
\partial_{j} T_{i j}=\varepsilon_{i k} j_{k} B
$$

and can be solved to yield for the current

$$
j^{i}=-\frac{1}{B} \varepsilon^{i j} \partial_{k} T_{j k}
$$

The equation for the conservation of charge is now

$$
\frac{\partial \rho}{\partial t}-\frac{1}{2 B} \partial_{i} \partial_{j}\left(\varepsilon_{i k} T_{k j}+\varepsilon_{j k} T_{i k}\right)=0
$$


One notices that the conservation law (44) is in the same form as (17) in the ealier version of the symmetric tensor gauge theory of fracton. Thus, the conservation of charge has a "higher-rank" form due to the fact that on the LLL the current density is no longer independent but can be expressed through the derivative of the stress tensor. The same conservation law was derived previously in Ref. [46] using a LLL field theory formalism. The connection of volume-preserving diffeomorphism and quantum Hall physics was also noticed in Refs. [14] and [26].

\section{B. The Wen-Zee term}

One possible term in the effective action for the fractional quantum Hall states is the Wen-Zee term [37]. To introduce this term, we need to define the Newton-Cartan geometry and through it the spin connection. We only give the relevant formulas here, for details see, e.g., Refs. $[44,47]$. The Newton-Cartan geometry structure is given by a one-form $n_{\mu}$ (in the simplest version of the geometry $d n=0$ ), a vector $v^{\mu}$, and a symmetric contravariant metric tensor $g^{\mu \nu}$ satisfying $n_{\mu} v^{\mu}=1, g^{\mu \nu} n_{\nu}=0$. In our case,

$$
n_{\mu}=(1, \mathbf{0}), \quad v^{\mu}=\left(\begin{array}{c}
1 \\
v^{i}
\end{array}\right), \quad g^{\mu \nu}=\left(\begin{array}{cc}
0 & 0 \\
0 & g^{i j}
\end{array}\right) .
$$

where

$$
v^{i}=\ell^{2} \varepsilon^{i j} \partial_{j} A_{0},
$$

and $g^{i j}$ is the matrix inverse of $g_{i j}$. One then defines the covariant metric tensor $g_{\mu \nu}$ so that $g_{\mu \nu} v^{\nu}=0$ and $g_{\mu \nu} g^{\nu \lambda}=\delta_{\mu}^{\lambda}-n_{\mu} v^{\lambda}$,

$$
g_{\mu \nu}=\left(\begin{array}{cc}
g_{i j} v^{i} v^{j} & -v_{j} \\
-v_{i} & g_{i j}
\end{array}\right),
$$

where $v_{i} \equiv g_{i j} v^{j}$, together with the Christoffel symbol which can be used to define covariant derivatives

$$
\Gamma_{\nu \lambda}^{\mu}=v^{\mu} \partial_{\nu} n_{\lambda}+\frac{1}{2} g^{\mu \rho}\left(\partial_{\nu} g_{\rho \lambda}+\partial_{\lambda} g_{\rho \nu}-\partial_{\rho} g_{\nu \lambda}\right) .
$$

One further defines the vielbein $e_{i}^{a}$ so that

$$
g^{\mu \nu}=e^{a \mu} e^{a \nu},
$$

and the spin connection is defined as

$$
\omega_{\mu}=\frac{1}{2} \varepsilon^{a b} e^{a \nu} \nabla_{\mu} e_{\nu}^{b}
$$

which, in components, reads

$$
\begin{aligned}
& \omega_{0}=\frac{1}{2}\left(\varepsilon^{a b} e^{a j} \partial_{0} e_{j}^{b}+\varepsilon^{i j} \partial_{i} v_{j}\right), \\
& \omega_{i}=\frac{1}{2}\left(\varepsilon^{a b} e^{a j} \partial_{i} e_{j}^{b}-\varepsilon^{j k} \partial_{j} g_{i k}\right) .
\end{aligned}
$$


The spin connection transforms like the gauge potential under the local $\mathrm{O}(2)$ rotation of the vielbein: $e^{a}(x) \rightarrow e^{a}(x)+\alpha(x) \varepsilon^{a b} e^{b}(x)$. The Wen-Zee term [37] is given by

$$
\frac{\kappa}{4 \pi} \varepsilon^{\mu \nu \lambda} \omega_{\mu} \partial_{\nu} A_{\lambda}=\frac{\kappa}{4 \pi}\left(\omega_{0} B+A_{0} \varepsilon^{i j} \partial_{i} \omega_{j}-\varepsilon^{i j} \omega_{i} \partial_{0} A_{j}\right)=\frac{\kappa}{4 \pi}\left(\frac{\omega_{0}}{\ell^{2}}+\frac{1}{2} A_{0} R\right),
$$

where we have put $\varepsilon^{i j} \partial_{i} A_{j}=\ell^{-2}$ and $\partial_{0} A_{i}=0$. The coefficient $\kappa$ is related to the filling fraction $\nu$ and the Wen-Zee shift $\mathcal{S}$ of a fraction quantum Hall (FQH) system by the relation

$$
\kappa=\nu \mathcal{S}
$$

Up to quadratic order and ignore the total total derivative terms, we can rewrite the Wen-Zee term as

$$
\frac{\kappa}{8 \pi}\left(A_{0} R-\frac{1}{4 \ell^{2}} \varepsilon^{i j} h_{i k} \dot{h}_{j k}\right),
$$

which is exactly the Chern-Simons term (15).

Some remarks are in order. We demonstrated that the famous Wen-Zee term in the literature of FQH is nothing but the Chern Simon term in the higher-rank gauge theory. One can think of this in the reversed order. The higher-rank gauge symmetry dictates the relation of the Wen-Zee shift $\mathcal{S}$ and the Hall viscosity $\eta_{H}$ which are determined by the two separated component of the Chern-Simons Lagrangian in the higher-rank gauge theory (55).

\section{GENERALIZATION TO (3+1) DIMENSIONS}

\section{A. Construction of the $(3+1) D$ nonlinear higher-rank symmetry}

In this seciton, we will generalize the nonabelian higher-rank symmetry to $(3+1)$ dimensions. To do that, we imagine a three-dimensional version of the LLL . Instead of a background magnetic field, we imagine a background Kalb-Ramond field. Concretely, we imagine a nonrelativistic theory living in background metric $g_{i j}$ and a Kalb-Ramond field $B_{\mu \nu}=-B_{\nu \mu}$. The field strength of the latter is

$$
H_{\mu \nu \lambda}=\partial_{\mu} B_{\nu \lambda}+\partial_{\nu} B_{\lambda \mu}+\partial_{\lambda} B_{\mu \nu}
$$

and we assume that there is gauge symmetry with one-form gauge parameter $\alpha_{\mu}$ under which

$$
\delta B_{\mu \nu}=\partial_{\mu} \alpha_{\nu}-\partial_{\nu} \alpha_{\mu}
$$

and $H_{\mu \nu \lambda}$ is invariant. Most crucially, we assume that our theory is invariant symmetry under time-dependent spatial diffeomorphisms,

$$
\begin{aligned}
\delta g_{i j} & =-\xi^{k} \partial_{k} g_{i j}-g_{k j} \partial_{i} \xi^{k}-g_{i k} \partial_{j} \xi^{k}, \\
\delta B_{i j} & =-\xi^{k} \partial_{k} B_{i j}-B_{k j} \partial_{i} \xi^{k}-B_{i k} \partial_{j} \xi^{k}, \\
\delta B_{i 0} & =-\xi^{k} \partial_{k} B_{i 0}-B_{k 0} \partial_{i} \xi^{k}-B_{i k} \dot{\xi}^{k} .
\end{aligned}
$$


which is $3 \mathrm{D}$ version of the the $m \rightarrow 0$ (i.e., LLL) limit the of the nonrelativistic diffeomorphism (34). We do not have a concrete example of a well-defined theory with the symmetry (58). As the LLL can be thought of as the massless limit for particles in a magnetic field, one can imagine a theory of massless strings coupled to a Kalb-Ramond field. The details (or even the existence) of such a theory is not important for our further discussion.

Following our discussion of the LLL in $(2+1) \mathrm{D}$, we assume that our system lives in a finite Kalb-Ramond field $H_{i j k}=\ell^{-3} \varepsilon_{i j k}$, and restrict ourselves to VPDs with $\partial_{k} \xi^{k}=0$ or

$$
\xi^{k}=\ell^{3} \varepsilon^{k l m} \partial_{l} \lambda_{m}
$$

The change of $B_{i j}$ under this VPD,

$$
\delta B_{i j}=-\ell^{3} \varepsilon^{k l m}\left(\partial_{k} B_{i j}+B_{k j} \partial_{i}+B_{i k} \partial_{j}\right) \partial_{l} \lambda_{m},
$$

again can be compensated by a gauge transformation (57) with the gauge parameter

$$
\alpha_{i}=\lambda_{i}-\ell^{3} \varepsilon^{k l m} B_{i k} \partial_{l} \lambda_{m} .
$$

The combined VPD and gauge transfomration changes only the $B_{0 i}$ components of the KalbRamond potential and the metric. This is a higher-rank symmetry which transforms our set of gauge fields $\left(B_{0 i}, g_{i j}\right)$ like

$$
\begin{aligned}
\delta B_{0 i} & =\dot{\lambda}_{i}-\ell^{3} \varepsilon^{k l m}\left(\partial_{k} B_{0 i}+B_{0 k} \partial_{i}\right) \partial_{l} \lambda_{m}, \\
\delta g_{i j} & =-\ell^{3} \varepsilon^{k l m}\left(\partial_{k} g_{i j}+g_{k j} \partial_{i}+g_{i k} \partial_{j}\right) \partial_{l} \lambda_{m} .
\end{aligned}
$$

In addition, we also inherit from the gauge symmetry (57) those transformations which leaves $B_{i j}$ invariant. These correspond to gauge parameters with vanishing spatial components: $\alpha_{\mu}=\left(\alpha_{0}, \mathbf{0}\right)$. Under these gauge transformations,

$$
\begin{aligned}
\delta B_{0 i} & =-\partial_{i} \alpha_{0}, \\
\delta g_{i j} & =0 .
\end{aligned}
$$

Equations (64) and (65) represent the full group of higher-rank symmetries. Those we have used an analogy with the LLL physics in 2D as a motivation, the resulting transformation laws do not require any LLL-type microscopic physics. In fact, we will see that the higherrank symmetry appears in the context of 3D ferromagnets.

As in $2 \mathrm{D}$, it is possible to "complete" $B_{0 i}$ by adding the spatial components $B_{i j}$ so that $B_{\mu \nu}$ form the set of Kalb-Ramond gauge potentials. This would make $B_{0 i}$ be the shared components of the two sets of gauge potentials: the Kalb-Ramond gauge potentials and the gauge potentials of the higher-rank symmetry of VPDs (see Fig. 2).

\section{B. Linearized higher-ranked symmetries and conservation laws}

At the linearlized level, the higher-rank gauge invariance is

$$
\begin{aligned}
\delta B_{0 i} & =\dot{\lambda}_{i}-\partial_{i} \alpha_{0}, \\
\delta h_{i j} & =-\ell^{3}\left(\varepsilon_{j k l} \partial_{i}+\varepsilon_{i k l} \partial_{j}\right) \partial_{k} \lambda_{l} .
\end{aligned}
$$




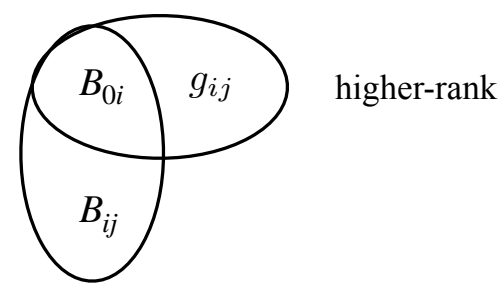

$U(1)$

FIG. 2. $B_{0 i}$ is shared by two sets of gauge potentials.

As far as we know, this linear higher-rank symmetry has not been considered previously in the literature.

Let us now assume that the currents coupled to $B_{0 i}$ and $h_{i j}$ be $J^{i}$ and $T^{i j}$,

$$
\delta \ln Z=\int d^{4} x\left(J^{i} \delta B_{0 i}+\frac{1}{2} T^{i j} \delta h_{i j}\right) .
$$

Two conservation laws follow from (66). First, from the gauge invariance with gauge parameter $\alpha_{0}$ implies that the "current" $J^{i}$ is divergence-free:

$$
\boldsymbol{\nabla} \cdot \mathbf{J}=0
$$

On the other hand the VPD invariance, generated by $\lambda_{i}$, leads to

$$
\frac{\partial J_{i}}{\partial t}-\ell^{3} \varepsilon_{i j k} \partial_{j} \partial_{l} T_{k l}=0
$$

This means the following quantities are conserved

$$
\begin{aligned}
\mathbf{I}_{1} & =\int d \mathbf{x} \mathbf{J}, \\
I_{2} & =\int d \mathbf{x}(\mathbf{x} \cdot \mathbf{J}), \quad \mathbf{I}_{3}=\int d \mathbf{x}(\mathbf{x} \times \mathbf{J}), \quad I_{4}^{i j}=\int d \mathbf{x} x^{\{i} J^{j\}}, \\
\mathbf{I}_{5} & =\int d \mathbf{x} \mathbf{x}(\mathbf{x} \cdot \mathbf{J}), \quad \mathbf{I}_{6}=\int d \mathbf{x} \frac{x^{2}}{2} \mathbf{J}, \\
I_{7}^{i j k} & =\int d \mathbf{x} x^{\{i} x^{j} J^{k\}} .
\end{aligned}
$$

On the other hand,

$$
I_{8}^{i j}=\int d \mathbf{x} x^{\{i}(\mathbf{x} \times \mathbf{J})^{j\}}
$$

is not conserved; its time derivative is proportional to $\int d \mathbf{x} T^{i j}$.

Assume that the "current" $\mathbf{J}$ is nozero only in a finite region of space, then because it is divergence-free, one can express it as the curl of a vector field: $\mathbf{J}=\boldsymbol{\nabla} \times \boldsymbol{\mu}$, where $\boldsymbol{\mu}$ vanishes at infinity. Then among the conserved quantities only the following are nonzero: $\mathbf{I}_{3}, \mathbf{I}_{5}$, and 
$\mathbf{I}_{6}$, and the last two quantities are not independent:

$$
\begin{aligned}
& \mathbf{I}_{3}=\int d \mathbf{x} \boldsymbol{\mu}, \\
& \mathbf{I}_{5}=-\mathbf{I}_{6}=\int d \mathbf{x}(\mathbf{x} \times \boldsymbol{\mu}) .
\end{aligned}
$$

One can think of $\mathbf{I}_{3}$ as the magnetic moment density and $\mathbf{J}$ as the magnetization current. Let us assume that there is a quasiparticle that carries a magnetic moment. Then the conservation of $\mathbf{I}_{3}$ means that the magnetic moment does not change its value. The conservation of $\mathbf{I}_{5}$ implies that a particle can move only along the direction of its magnetic moment, but not along the two perpendicular directions.

We will show in Section VID that the symmetry described here is realized in the nonlinear sigma model describing 3D ferromagnets.

\section{EXAMPLES OF THEORIES WITH VOLUME-PRESERVING DIFFEOMORPHISM INVARIANCE}

For the abelian higher-rank symmetry, one of the simplest ways to couple a matter field to the gauge field $\left(A_{0}, h_{i j}\right)$ is to introduce a Goldstone boson $\varphi$ which tranforms under the gauge transformation as $\varphi \rightarrow \varphi+\lambda$ and write

$$
\mathcal{L}=\frac{c_{1}}{2}\left(\partial_{0} \varphi-A_{0}\right)^{2}-\frac{c_{2}}{2}\left(\partial_{i} \partial_{j} \varphi-h_{i j}\right)^{2} .
$$

However, we were not able to find a nonlinear version of this transformation. For example, if one postulates

$$
\delta_{\lambda} \varphi=\lambda-\ell^{2} \varepsilon^{k l} \partial_{k} \varphi \partial_{l} \lambda,
$$

then one can check by direct calculation that $\left[\delta_{\alpha}, \delta_{\beta}\right] \varphi \neq \delta_{[\alpha, \beta]} \varphi$, so such transformation law would be inconsistent. We have to devise other ways to couple matter to the gauge field.

\section{A. Composite fermions}

In the fractional quantum Hall effect at filling fractions $\nu=1 / 2, \nu=1 / 4$ etc., the quasiparticle is electrically neutral. One can consistently couple such a particle to the higherrank gauge field. For example, a Lagrangian for a nonrelativistic particle with dispersion relation $\omega=k^{2} / 2 m$ would be

$$
L=\frac{i}{2} v^{\mu} \psi^{\dagger} \stackrel{\leftrightarrow}{\partial}_{\mu} \psi-\frac{1}{2 m} g^{\mu \nu} \partial_{\mu} \psi^{\dagger} \partial_{\nu} \psi=\frac{i}{2} \psi^{\dagger} \stackrel{\leftrightarrow}{\partial}_{0} \psi+\frac{i}{2} \ell^{2} \varepsilon^{i j} \partial_{j} A_{0} \psi^{\dagger} \stackrel{\leftrightarrow}{\partial}_{i} \psi-\frac{g^{i j}}{2 m} \partial_{i} \psi^{\dagger} \partial_{j} \psi .
$$

One can interpret the coupling of the electric field $\partial_{i} A_{0}$ with the particle momentum as a dipole moment, perpendicular to the direction of its motion. This is consistent with the constraints that follow from conservation laws. In fact, that is how a composite fermion in the fractional quantum Hall state at $\nu=1 / 2$ or $\nu=1 / 4$ should couple to the external potential: the composite fermion is neutral but possesses an electric dipole moment. 


\section{B. Crystal on the lowest Landau level}

Another way to realize the higher-rank symmetry is through an effective theory of a solid. Such a solid may be realized as a Wigner crystal, which is expected to be the ground state of electrons on the LLL at small filling fractions. A solid is parametrized by a map from the physical coordinates $x^{i}$ to the coordinate system $X^{a}$ frozen into the solid: $X^{a}=X^{a}\left(x^{i}\right)$ [48]. In the ground state $X^{a}=x^{i} \delta_{a i}$, the displacement $u^{a}$ are defined as $X^{a}=x^{a}-u^{a}$. On the LLL the coordinates of a lattice site do not commute: $[x, y]=-i \ell^{2}$. The Lagrangian thus should contain the following term

$$
S_{\text {Berry }}=\frac{n_{0}}{2 \ell^{2}} \varepsilon^{a b} X^{a} \partial_{t} X^{b},
$$

where $n_{0}$ is the equilibrium particle number density. Under a VPD $X^{a}$ transforms as

$$
\delta_{\lambda} X^{a}=-\ell^{2} \varepsilon^{i j} \partial_{i} X^{a} \partial_{j} \lambda,
$$

and so

$$
\delta_{\lambda} S_{\text {Berry }}=-\frac{n_{0}}{2} \varepsilon^{i j} \varepsilon^{a b} X^{a} \partial_{i} X^{b} \partial_{j} \dot{\lambda}=-\frac{n_{0}}{2} \dot{\lambda} \varepsilon^{i j} \varepsilon^{a b} \partial_{i} X^{a} \partial_{j} X^{b} .
$$

This change of the action can be compensated by including a term proportional to $A_{0}$ into the Lagrangian. The full Lagrangian is then

$$
\mathcal{L}=\frac{n_{0}}{2 \ell^{2}} \varepsilon^{a b} X^{a} \partial_{t} X^{b}+\frac{n_{0}}{2} A_{0} \varepsilon^{a b} \varepsilon^{i j} \partial_{i} X^{a} \partial_{j} X^{b}-\varepsilon\left(O^{a b}\right),
$$

with $O^{a b}=g^{i j} \partial_{i} X^{a} \partial_{j} X^{b}$ and $\varepsilon\left(O^{a b}\right)$ is the energy associated with elastic deformations. The spectrum of this theory can be obtained by expanding the action to quadratic order over the displacement $u^{a}$. The presence of a term with first time derivative implies that the dispersion relation of the lattice sound wave has the quadratic form $\omega \sim q^{2}$ rather than the linear form.

Thus, we have been able to construct the effective theory of a Wigner crystal on the LLL starting from the higher-rank symmetry.

\section{Vortex crystal}

Another type of matter is the "vortex crystal," which is realized, for example, in a rotating Bose gas [49]. The crystal is formed by the zeros of the condensate wave function. In this case, the lattice fields $X^{a}$ do not couple to $A_{0}$ directly, but through a dynamical gauge field $a_{\mu}$, which is the dual of the superfluid phonon. Under VPD $a_{\mu}$ transforms as a one-form,

$$
\delta a_{\mu}=-\ell^{2} \varepsilon^{k l}\left(\partial_{k} a_{\mu}-a_{k} \partial_{\mu}\right) \partial_{l} \lambda .
$$

The field $a_{\mu}$ is the coupled to the background $A_{0}$ through the following term

$$
\frac{1}{2 \pi} \int d^{3} x\left(\frac{a_{0}}{\ell^{2}}+A_{0} b\right) \text {. }
$$


One can check directly that this term is invariant under VPDs. It is also obviously invariant under gauge transformations $a_{\mu} \rightarrow a_{\mu}+\partial_{\mu} \alpha$. In fact, (86) can be obtained from the ChernSimons term $\frac{1}{2 \pi} \varepsilon^{\mu \nu \lambda} a_{\mu} \partial_{\nu} A_{\lambda}$ by putting $A_{i}$ to be a static background with $\varepsilon^{i j} \partial_{i} A_{j}=\ell^{-2}$.

The Lagriangian of the vortex crystal is then determined by the symmetry and reads

$$
\mathcal{L}=-\varepsilon^{\mu \nu \lambda} \varepsilon^{a b} a_{\mu} \partial_{\nu} X^{a} \partial_{\lambda} X^{b}-\varepsilon\left(O^{a b}\right)-\varepsilon_{b}(b)+\frac{1}{2 \pi}\left(\frac{a_{0}}{\ell^{2}}+A_{0} b\right),
$$

where $\varepsilon\left(O^{a b}\right)$ and $\varepsilon_{b}(b)$ represent the energies of the lattice and of the condensate, respectively. Assuming $a_{\mu}$ transforms like a one-form under VPD, it can be checked that the Lagrangian above is invariant with respect to this symmetry. The eigenmode of this theory is again a Tkachenko mode with a quadratic dispersion relation [49].

Note that the above Lagrangian contains only leading-derivative terms and does not include next-to-leading terms considered in Ref. [49].

\section{Ferromagnets}

We now show that the ferromagnet in $2+1 \mathrm{D}$ secretly possesses a higher-rank symmetry similar to the models with particles on the LLL ${ }^{2}$. At the long-wavelength limit, a ferromagnet is described by a nonlinear sigma model, written in terms of an $\mathrm{O}(3)$ unit vector $n^{a}, n^{a} n^{a}=1$, with the action

$$
S=S_{\text {Berry }}+S_{\mathrm{NLS}}=S_{0} \int_{0}^{1} d \sigma \int d t d \mathbf{x} \varepsilon^{a b c} n^{a} \partial_{t} n^{b} \partial_{\sigma} n^{c}-\frac{J}{2} \int d t d \mathbf{x} \delta^{i j} \partial_{i} n^{a} \partial_{j} n^{a} .
$$

The first term is a Wess-Zumino topological term in spin's action, which is induced by the Berry phase [51]. The second term is the energy term of the nonlinear sigma model and can made invariant under VPD by replacing $\delta^{i j}$ with $g^{i j}$. The first term is however not invariant:

$$
\delta_{\lambda} S_{\text {Berry }}=-S_{0} \ell^{2} \int_{0}^{1} d \sigma \int d t d \mathbf{x} \varepsilon^{a b c} \varepsilon^{i j} n^{a} \partial_{i} n^{b} \partial_{\sigma} n^{c} \partial_{j} \dot{\lambda}
$$

Integrating by parts, taking into account that $\epsilon^{a b c} \partial_{j} n^{a} \partial_{i} n^{b} \partial_{\sigma} n^{c}=0$ (this is because all the three $\mathrm{O}(3)$ vectors $\partial_{i} n^{a}, \partial_{j} n^{a}, \partial_{\sigma} n^{a}$ are perpendicular to $n^{a}$ and hence are linearly dependent) we find

$$
\begin{aligned}
\delta_{\lambda} S_{\text {Berry }} & =S_{0} \ell^{2} \int_{0}^{1} d \sigma \int d t d \mathbf{x} \varepsilon^{a b c} \varepsilon^{i j} n^{a} \partial_{i} n^{b} \partial_{j} \partial_{\sigma} n^{c} \dot{\lambda} \\
& =\frac{S_{0}}{2} \ell^{2} \int_{0}^{1} d \sigma \int d t d \mathbf{x} \varepsilon^{a b c} \varepsilon^{i j} \partial_{\sigma}\left(n^{a} \partial_{i} n^{b} \partial_{j} n^{c}\right) \dot{\lambda}=\frac{S_{0}}{2} \ell^{2} \int d t d \mathbf{x} \varepsilon^{a b c} \varepsilon^{i j} n^{a} \partial_{i} n^{b} \partial_{j} n^{c} \dot{\lambda} .
\end{aligned}
$$

\footnotetext{
${ }^{2}$ In fact, one can show that the dynamical equation of a single skyrmion in a 2-dimensional ferromagnet is the same as the equation of motion of a charged particle in a constant magnetic field [50].
} 
We now choose

$$
\ell^{2}=\frac{1}{4 \pi S_{0}}
$$

add the following term to the action

$$
S_{A_{0}}=-\frac{1}{8 \pi} \int d t d \mathbf{x} A_{0} \varepsilon^{a b c} \varepsilon^{i j} n^{a} \partial_{i} n^{b} \partial_{j} n^{c} .
$$

Then $\delta_{\lambda}\left(S_{\text {Berry }}+S_{A_{0}}\right)=0$. Thus if we couple the ferromagnetic order parameter with the gauge fields $A_{0}, g_{i j}$ in the following way

$$
S=S_{\text {Berry }}+S_{A_{0}}-\frac{f^{2}}{2} \int d t d \mathbf{x} g^{i j} \partial_{i} n^{a} \partial_{j} n^{a},
$$

then the action is invariant under VPD with $\ell^{2}$ defined in Eq. (91).

In fact, one can further introduce into the theory the vector gauge potential $A_{i}$, promoting Eq. (92) to

$$
S_{A_{\mu}}=-\frac{1}{8 \pi} \int d t d \mathbf{x} A_{\mu} \varepsilon^{a b c} \varepsilon^{\mu \nu \lambda} n^{a} \partial_{\nu} n^{b} \partial_{\lambda} n^{c} .
$$

with $A_{i}$ transforming as a one-form under VPDs. In this case the potential $A_{0}$ is simultaneously the scalar component of the $\mathrm{U}(1)$ gauge field $\left(A_{0}, A_{i}\right)$ and the scalar component of the gauge potential of a higher-spin symmetry, $\left(A_{0}, g_{i j}\right)$ (Fig. 1).

Now the scalar potential $A_{0}$ is coupled to the topological charge density $\rho(\mathbf{x})$. This means that a skyrmion behaves like a particle in an effective magnetic field with magnitude

$$
B_{\text {eff }}=-4 \pi S_{0} q
$$

where $q=\int d \mathbf{x} \rho(\mathbf{x})$ is the topological charge of the skyrmion. This fact can be derived by calculating the Berry phase associated with the motion of a skyrmion [39]. One also finds that the following quantities

$$
\int d \mathbf{x} \rho, \quad \int d \mathbf{x} x^{i} \rho, \quad \int d \mathbf{x} x^{2} \rho
$$

are conserved. This fact is again well known [38]. ${ }^{3}$

For a ferromagnet in $(3+1) \mathrm{D}$, the term $S_{A_{0}}$ that couples $A_{0}$ to the topological charge density is replaced by the coupling of $B_{0 i}$ to the density of a one-form current,

$$
S_{B_{0 i}}=-\frac{1}{8 \pi} \int d t d \mathbf{x} \varepsilon^{a b c} \varepsilon^{i j k} B_{0 i} n^{a} \partial_{j} n^{b} \partial_{k} n^{c},
$$

and this has the $(3+1) \mathrm{D}$ version of VPD invariance with $\ell^{3}=\left(4 \pi S_{0}\right)^{-1}$. Again one can promote $(97)$ to

$$
S_{B_{\mu \nu}}=-\frac{1}{8 \pi} \int d t d \mathbf{x} \varepsilon^{a b c} \varepsilon^{\mu \nu \lambda \rho} B_{\mu \nu} n^{a} \partial_{\lambda} n^{b} \partial_{\rho} n^{c}
$$

\footnotetext{
${ }^{3}$ In the presence of the Dyaloshinskii-Morya interaction, which breaks the higher-rank symmetry, only the first two quantities are conserved [52].
} 
In this case, $B_{0 i}$ are the shared components of a Kalb-Ramond gauge field and the set of gauge potentials of a higher-rank gauge symmetry $\left(B_{0 i}, g_{i j}\right)$ (see Fig. 2).

It was found in Ref. [38] that $\mathbf{I}_{3}$ and $\mathbf{I}_{5}=-\mathbf{I}_{6}$ are conserved. We have given this fact a new interpretation in terms of a hidden higher-rank symmetry.

It is instructive to rewrite the ferromagnet in the $\mathbb{C P}^{1}$ parametrization, where

$$
n^{a}=z^{\dagger} \sigma^{a} z, \quad z=\left(\begin{array}{c}
z_{1} \\
z_{2}
\end{array}\right), \quad z^{\dagger} z=1 .
$$

The action of the ferromagnet is then [50]

$$
S=S_{B e r r y}+S_{N L S}=2 i S_{0} \int d t d \mathbf{x} z^{\dagger} \partial_{t} z-2 J \int d t d \mathbf{x} D_{i} z^{\dagger} D_{i} z,
$$

where $D_{i} z \equiv\left(\partial_{i}-i a_{i}\right) z$, and $a_{i}$ is promoted to a dynamical field.

Now we couple the $\mathbb{C P}^{1}$ model to the external probes $\left(g_{i j}, A_{0}\right)$. We assume that under $\mathrm{VPD} z$ transforms as

$$
\delta_{\lambda} z=-\ell^{2} \varepsilon^{k l} \partial_{k} z \partial_{l} \lambda
$$

therefore,

$$
\delta_{\lambda} S_{\text {Berry }}=-2 i S_{0} \ell^{2} \int d t d \mathbf{x} \varepsilon^{i j} z^{\dagger} \partial_{i} z \partial_{j} \dot{\lambda}=-2 i S_{0} \ell^{2} \int d t d \mathbf{x} \dot{\lambda} \varepsilon^{i j} \partial_{i} z^{\dagger} \partial_{j} z
$$

We now add the following term to the action:

$$
S_{A_{0}}=2 i S_{0} \ell^{2} \int d t d \mathbf{x} A_{0} \varepsilon^{i j} \partial_{i} z^{\dagger} \partial_{j} z
$$

Then $\delta_{\lambda}\left(S_{B e r r y}+S_{A_{0}}\right)=0$, therefore the coupling of the ferromagnetic model with the gauge field $g_{i j}, A_{0}$ is

$$
S=S_{\text {Berry }}+S_{A_{0}}-2 J \int d t d \mathbf{x} g^{i j} D_{i} z^{\dagger} D_{j} z
$$

and respects the higher-rank symmetry.

\section{CONCLUSION}

We have presented a nonlinear version of a higher-rank gauge symmetry. The symmetry is basically that of volume-preserving diffeomorphism. We show several examples of coupling of matter with the higher-rank gauge potential that respects the symmetry. Many examples are taken from the physics of the LLL, which we show to naturally have volume-preserving diffeomorphism invariance. We also show that the nonlinear model of ferromagnetism also exhibits this symmetry, if one couples a gauge potential of the higher-rank symmetry with the topological charge density.

We have shown that, under certain condition, the charge densities satisfy the commutation relation of the VPD, i.e., the $w_{\infty}$ algebra. In this way one can easily understand 
why this algebra is realized in the bimetric model of FQH effect [53], without doing explicit calculations. One interesting question is whether the $w_{\infty}$ symmetry can be "upgraded" to the quantum $W_{\infty}$ symmetry $[33,34]$. This question, relevant for the fractional quantum Hall effect, is deferred to future work.

The authors thank Adrey Gromov and Sergej Moroz for discussions and comments on the earlier draft of this paper. This work is supported, in part, by the U.S. DOE grant No. DE-FG02-13ER41958, a Simons Investigator grant and by the Simons Collaboration on Ultra-Quantum Matter, which is a grant from the Simons Foundation (651440, DTS). DXN is supported by the Brown Theoretical Physics Center.

[1] M. Pretko, Subdimensional particle structure of higher rank $U(1)$ spin liquids, Phys. Rev. B 95, 115139 (2017), arXiv:1604.05329.

[2] M. Pretko, Generalized electromagnetism of subdimensional particles: A spin liquid story, Phys. Rev. B 96, 035119 (2017), arXiv:1606.08857.

[3] K. Slagle and Y. B. Kim, Quantum field theory of X-cube fracton topological order and robust degeneracy from geometry, Phys. Rev. B 96, 195139 (2017), arXiv:1708.04619.

[4] A. Prem, M. Pretko, and R. M. Nandkishore, Emergent phases of fractonic matter, Phys. Rev. B 97, 085116 (2018), arXiv:1709.09673.

[5] Z. Zhai and L. Radzihovsky, Fractonic gauge theory of smectics, (2020), arXiv:2012.02208.

[6] J. Wang and S.-T. Yau, Non-abelian gauged fracton matter field theory: Sigma models, superfluids, and vortices, Phys. Rev. Research 2, 043219 (2020), arXiv:1912.13485.

[7] T. Brauner, Field theories with higher-group symmetry from composite currents, (2021), arXiv:2012.00051.

[8] O. Dubinkin, A. Rasmussen, and T. L. Hughes, Higher-form gauge symmetries in multipole topological phases, Ann. Phys. (N.Y.) 422, 168297 (2020), arXiv:2007.05539.

[9] M. Qi, L. Radzihovsky, and M. Hermele, Fracton phases via exotic higher-form symmetrybreaking, Ann. Phys. (N.Y.) 424, 168360 (2021), arXiv:2010.02254.

[10] N. Seiberg, Field theories with a vector global symmetry, SciPost Phys. 8, 050 (2020), arXiv:1909.10544.

[11] N. Seiberg and S.-H. Shao, Exotic symmetries, duality, and fractons in 2+1-dimensional quantum field theory, SciPost Phys. 10, 027 (2021), arXiv:2003.10466.

[12] R. M. Nandkishore and M. Hermele, Fractons, Annu. Rev. Condens. Matter Phys. 10, 295-313 (2019), arXiv:1803.11196.

[13] A. Gromov, Towards classification of fracton phases: The multipole algebra, Phys. Rev. X 9, 031035 (2019), arXiv:1812.05104.

[14] A. Gromov, Chiral topological elasticity and fracton order, Phys. Rev. Lett. 122, 076403 (2019), arXiv:1712.06600.

[15] C. Chamon, Quantum glassiness in strongly correlated clean systems: An example of topo- 
logical overprotection, Phys. Rev. Lett. 94, 040402 (2005), cond-mat/0404182.

[16] S. Bravyi, B. Leemhuis, and B. M. Terhal, Topological order in an exactly solvable 3d spin model, Ann. Phys. (N.Y.) 326, 839 (2011), arXiv:1006.4871.

[17] J. Haah, Local stabilizer codes in three dimensions without string logical operators, Phys. Rev. A 83, 042330 (2011), arXiv:1101.1962.

[18] S. Vijay, J. Haah, and L. Fu, A new kind of topological quantum order: A dimensional hierarchy of quasiparticles built from stationary excitations, Phys. Rev. B 92, 235136 (2015), arXiv:1505.02576.

[19] M. Pretko, X. Chen, and Y. You, Fracton phases of matter, Int. J. Mod. Phys. A 35, 2030003 (2020), arXiv:2001.01722.

[20] M. Pretko and L. Radzihovsky, Fracton-Elasticity Duality, Phys. Rev. Lett. 120, 195301 (2018), arXiv:1711.11044.

[21] V. Cvetkovic, Z. Nussinov, and J. Zaanen, Topological kinematic constraints: dislocations and the glide principle, Philosophical Magazine 86, 2995 (2006).

[22] A. J. Beekman, J. Nissinen, K. Wu, K. Liu, R.-J. Slager, Z. Nussinov, V. Cvetkovic, and J. Zaanen, Dual gauge field theory of quantum liquid crystals in two dimensions, Physics Reports 683, 1 (2017).

[23] H. Kleinert, Duality transformation for defect melting, Physics Letters A 91, 295 (1982).

[24] H. Kleinert, Dual model for dislocation and disclination melting, Physics Letters A 96, 302 (1983).

[25] M. Pretko and L. Radzihovsky, Symmetry Enriched Fracton Phases from Supersolid Duality, Phys. Rev. Lett. 121, 235301 (2018), arXiv:1808.05616.

[26] D. Doshi and A. Gromov, Vortices as fractons, Commun. Phys. 4, 44 (2021), arXiv:2005.03015.

[27] D. X. Nguyen, A. Gromov, and S. Moroz, Fracton-elasticity duality of two-dimensional superfluid vortex crystals: defect interactions and quantum melting, SciPost Phys. 9, 076 (2020), arXiv:2005.12317.

[28] L. Radzihovsky, Quantum Smectic Gauge Theory, Phys. Rev. Lett. 125, 267601 (2020), arXiv:2009.06632.

[29] J. Wang and K. Xu, Higher-rank tensor field theory of non-abelian fracton and embeddon, Ann. Phys. (N.Y.) 424, 168370 (2021), arXiv:1909.13879.

[30] J. Wang, K. Xu, and S.-T. Yau, Higher-rank tensor non-abelian field theory: Higher-moment or subdimensional polynomial global symmetry, algebraic variety, noether's theorem, and gauging, Phys. Rev. Research 3, 013185 (2021).

[31] J. Wang and S.-T. Yau, Non-abelian gauged fracton matter field theory: Sigma models, superfluids, and vortices, Phys. Rev. Research 2, 043219 (2020).

[32] S. Girvin, A. MacDonald, and P. Platzman, Magneto-roton theory of collective excitations in the fractional quantum Hall effect, Phys. Rev. B 33, 2481 (1986).

[33] A. Cappelli, C. A. Trugenberger, and G. R. Zemba, Infinite symmetry in the quantum Hall effect, Nucl. Phys. B 396, 465 (1993), arXiv:hep-th/9206027. 
[34] S. Iso, D. Karabali, and B. Sakita, Fermions in the lowest Landau level: Bosonization, $W_{\infty}$ algebra, droplets, chiral bosons, Phys. Lett. B 296, 143 (1992), arXiv:hep-th/9209003.

[35] A. Cappelli and E. Randellini, Multipole expansion in the quantum Hall effect, J. High Energy Phys. 2016, 105, arXiv:1512.02147.

[36] D. X. Nguyen, S. Golkar, M. M. Roberts, and D. T. Son, Particle-hole symmetry and composite fermions in fractional quantum hall states, Phys. Rev. B 97, 195314 (2018), arXiv:1709.07885.

[37] X. Wen and A. Zee, Shift and Spin Vector: New Topological Quantum Numbers for the Hall Fluids, Phys. Rev. Lett. 69, 953 (1992), [Erratum: Phys. Rev. Lett. 69, 3000 (1992)].

[38] N. Papanicolaou and T. Tomaras, Dynamics of magnetic vortices, Nucl. Phys. B 360, 425 (1991).

[39] M. Stone, Magnus force on skyrmions in ferromagnets and quantum Hall systems, Phys. Rev. B 53, 16573 (1996).

[40] P. A. M. Dirac, Generalized Hamiltonian Dynamics, Can. J. Math. 2, 129 (1950).

[41] M. Pretko, Higher-spin Witten effect and two-dimensional fracton phases, Phys. Rev. B 96, 125151 (2017), arXiv:1707.03838.

[42] Y. You, T. Devakul, S. L. Sondhi, and F. J. Burnell, Fractonic Chern-Simons and BF theories, Phys. Rev. Research 2, 023249 (2020), arXiv:1904.11530.

[43] C. Xu, Novel algebraic boson liquid phase with soft graviton excitations, (2006), arXiv:condmat/0602443.

[44] M. Geracie, D. T. Son, C. Wu, and S.-F. Wu, Spacetime symmetries of the quantum Hall effect, Phys. Rev. D 91, 045030 (2015), arXiv:1407.1252.

[45] C. Hoyos and D. T. Son, Hall Viscosity and Electromagnetic Response, Phys. Rev. Lett. 108, 066805 (2012), arXiv:1109.2651.

[46] D. X. Nguyen, D. T. Son, and C. Wu, Lowest Landau Level Stress Tensor and Structure Factor of Trial Quantum Hall Wave Functions (2014), arXiv:1411.3316.

[47] D. T. Son, Newton-Cartan Geometry and the Quantum Hall Effect, (2013), arXiv:1306.0638.

[48] D. E. Soper, Classial Field Theory (Wiley, New York, NY, 1976).

[49] S. Moroz and D. T. Son, Bosonic Superfluid on the Lowest Landau Level, Phys. Rev. Lett. 122, 235301 (2019), arXiv:1901.06088.

[50] J. H. Han, Skyrmions in Condensed Matter (Springer, Cham, Switzerland, 2017).

[51] E. Fradkin, Field Theories of Condensed Matter Physics, 2nd ed. (Cambridge University Press, Cambridge, 2013).

[52] S. Komineas and N. Papanicolaou, Skyrmion dynamics in chiral ferromagnets, Phys. Rev. B 92, 064412 (2015), arXiv:1505.04377.

[53] A. Gromov and D. T. Son, Bimetric Theory of Fractional Quantum Hall States, Phys. Rev. X 7, 041032 (2017), [Addendum: Phys. Rev. X 8, 019901 (2018)], arXiv:1705.06739. 